\title{
Plan integral de mantenimiento preventivo en las instalaciones del museo arte contemporaneo-maczul
}

\author{
L. Ramírez ${ }^{1}$, M. Sánchez², A. Perozo ${ }^{3}$ \\ ${ }^{1}$ Otepi Consultores S.A. Zona Rental de la Universidad Metropolitana, Caracas. \\ ${ }^{2}$ Centro de Estudios de Corrosión-Universidad del Zulia-Maracaibo-Venezuela. \\ ${ }^{3}$ Gerencia de mantenimiento, Posgrado de Ingeniería-Universidad del Zulia.
}

\section{Información del artículo \\ DOI: \\ http://dx.doi.org/10.21041/ra.v1 \\ i1.6 \\ Artículo recibido el 31 de \\ Agosto de 2010, revisado bajo \\ las politicas de publicación de \\ la Revista ALCONPAT y \\ aceptado el 01 de Diciembre de \\ 2010. Cualquier discusión, \\ incluyendo la réplica de los \\ autores se publicará en el tercer \\ número del año siempre y \\ cuando la información se reciba \\ antes del cierre del segundo \\ número del año 2011}

\section{(C) 2011 Alconpat Internacional}

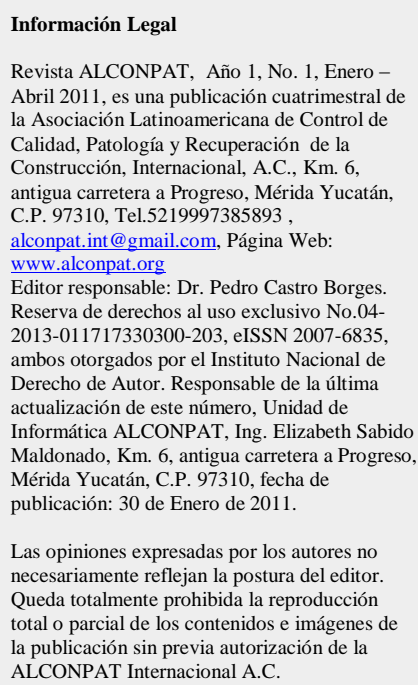

\section{RESUMEN}

La necesidad de preservación de las instalaciones de un museo está unida al atractivo propio del mismo y debe reflejar en todo sentido la naturaleza de la función para la cual fue creado. Para ello se debe tener en cuenta una serie de variables y parámetros según las particulares y exigencias que tienen las edificaciones de los museos, al prestar el servicio para el cual han sido destinados, así como sus elementos más relevantes en cuanto infraestructura y servicios, que va más allá del simple buen estado y funcionamiento de los mismos. En este sentido se ha preparado las bases metodológicas de un modelo de integral mantenimiento para el museo de arte contemporáneo del Zulia (MACZUL) que incluya tanto la preservación de la apariencia física externa de las instalaciones del museo, de los sistemas y bienes que sirvan de apoyo a su labor, así como lo referente al resguardo de las áreas de exposición de las obras, según la naturaleza y materiales de que están elaboradas, de manera que permanezcan en el mejor estado de conservación. En los servicios que se brindan al público, se debe usar las bases de datos como herramienta auxiliar de soporte para el manejo y gestión de la información. El desarrollo de este modelo y la herramienta de apoyo propuesta permiten formular un sistema de manejo de información, suficientemente ágil y flexible, con la aplicación y uso las bases de datos como herramienta básica de soporte para construir la estructura administrativa de gestión de la información del plan de mantenimiento del museo junto a las tareas propias del proceso y estructura del mantenimiento. Un aspecto adicional que amerita particular atención son las condiciones ambientales particularmente críticas a la cual está expuesta la estructura externa del MACZUL, las cuales añaden y promueven un alto grado de corrosión.

Palabras clave: museos; preservación; mantenimiento preventivo integral; estructura administrativa de gestión de información; bases de datos.

\begin{abstract}
The preservation of infrastructure for museums must consider the specific conditions and needs during the service life of the building. This works shows the preparation of the methodological basis for an integral model for the maintenance of the Contemporary Art Museum of Zulia (MACZUL), which includes the preservation of the external aesthetic features, the systems that support its functions, and the exhibition areas. The development of the model and the proposed tool of support allows the formulation of an agile system to set up an administrative structure for the information management for the maintenance plan. The environmental conditions require special attention with regards to the soundness of the structure, as these favor corrosion process. Key words: Museums, preservation, integral preventive maintenance, administrative structure for information management, databases.
\end{abstract}

Autor de contacto: Miguel Sánchez (miguelcvc@yahoo.com) 
Revista ALCONPAT, Volumen 1, Número 1, Enero-Abril 2011, Páginas 50 - 63

\section{INTRODUCCION}

Los museos de arte son instituciones de carácter artístico y cultural destinados a ofrecer a la colectividad un lugar adecuado y un ambiente idóneo que permita y facilite el intercambio al público en general las diversas manifestaciones artísticas, con sus principales exponentes y representantes y sus trabajos más relevantes en las diversas ramas de la pictografía, escultura, arte folklórico, tradicional o de vanguardia, tanto nacional como internacional (Lucena 1999).

La naturaleza y funciones propias de las instalaciones de un museo deben estar unidas y en armonía al atractivo propio de las obras que el mismo debe mostrar. Debe reflejar, en todo momento tanto en lo externo como distribución funcional interna, la naturaleza y la función para la cual ha sido creado: La exposición y preservación de los bienes culturales y las obras del patrimonio artístico y cultural de una nación.

Esta ambición exige (como en toda edificación) diseñar y elaborar un plan de mantenimiento y cuidado que integre tanto la apariencia física externa de las instalaciones del museo, sistemas y bienes inmobiliarios que sirven de apoyo a su labor, servicios que ha de brindar al público así como de los sistemas y equipos responsables del control de las condiciones ambientales de luz, temperatura, humedad, seguridad y acondicionamiento de las áreas de exposición y almacenamiento de las obras según la naturaleza y tipo de materiales, en que están elaboradas y a las que son las responsables de garantizar el ambiente idóneo, para la permanencia y preservación de las colecciones en el mejor estado de conservación durante el mayor tiempo posible (CONAC 1992, Provenzali 2000).

Estas condiciones óptimas del ambiente (humedad, temperatura, nivel de exposición a la luz, control de plagas, etc.) son de particular importancia para la misión que cumplen los museos en las diversas áreas del museo tanto en las áreas administrativas, como en las de exposición, depósito, laboratorios y/o talleres de tratamiento y preservación etc. Por otro lado, nunca se insistirá demasiado en la necesidad de sensibilizar a la sociedad para la conservación de su patrimonio artístico cultural, sea este el propio de la nación o cualquier otro más allá de sus fronteras. La sensibilización en este legado permitirá apreciar con mayor fuerza las experiencias que enriquecen la identidad, y los valores de la cultura de un pueblo, que son luego la base sobre la cual se sustentarán logros y realizaciones históricas futuras. Adicionalmente, el estado de conservación del patrimonio cultural es en esencia el testimonio principal de vitalidad de la memoria y la identidad de la nación. El desarrollo y la evolución por una parte de una legislación para su salvaguarda el desarrollo institucional correspondiente y la participación de la ciudadanía en su preservación, expresan la evolución cultural y cívica de la población y por consiguiente su nivel de conciencia acerca de su significado y valor en el desarrollo del país (Consejo Internacional de Museos 1993).

Este trabajo trata del interés, por enmarcar los planteamientos hechos dentro del ámbito actual de atención a las instituciones, que tienen como tarea la difusión educativa del patrimonio artístico y cultural como son los Museos, y en particular del Museo de Arte Contemporáneo del Zulia (MACZUL), quien debe aportar un esquema de trabajo, que racionalmente ayude a la preservación en el mayor tiempo posible de las instalaciones del Museo y por ende de las colecciones artísticas bajo su custodia. 
Revista ALCONPAT, Volumen 1, Número 1, Enero-Abril 2011, Páginas 50 - 63

\section{PROCEDIMIENTO EXPERIMENTAL}

\subsection{Consideraciones de diseño de la base de datos para el Plan de Mantenimiento del Museo}

A través del presente estudio se realizan diversos planteamientos que en principio están dirigidos para la elaboración de un Plan de Mantenimiento de perspectiva amplia, que se puede ajustar en primer lugar a las condiciones y peculiaridades de cualquier Museo, usando como referencia al MACZUL, por ser este un ejemplo que reúne en si mismo todas los servicios que exige una edificación de tal naturaleza, con todos sus complejos aspectos y requerimientos de índole funcional y operativa, y que puede ser extensivo a cualquier Museo, que sea similar o más modesto en su infraestructura y de menor alcance en los servicios que presta (Fundación MACZUL 2000, MACZUL 1999).

Sin embargo, las propuestas señaladas en este trabajo distan mucho de cubrir la gran cantidad de aspectos puntuales y particulares que aún deben ser incorporados en el Plan de Mantenimiento Integral. El mismo soporte de gestión de la información propuesta, es decir la base de datos, exigirá una continua revisión para irse adaptando a estas particularidades que inevitablemente se presentarán durante el uso e implementación del plan de mantenimiento (Morrow 1984, Nava 1996, Perozo, A. 1999, Figueroa et.al., 2002, Stewart 1968).

En la elaboración de las tablas, debe estar muy unido un conocimiento exacto de las relaciones que existen en los equipos, sistemas, áreas, recursos humanos y materiales, así como de los costos asociados y el financiamiento disponible, que garantice el éxito final de su implementación, al margen de las consideraciones de índole político-cultural a la cual deben enfrentarse las iniciativas culturales en un país como el nuestro.

En el caso de la especificación clara y precisa de los diversos modos en que se dan las relaciones entre los sistemas y componentes del Museo, cabe destacar, que la determinación de estas relaciones deben seguir los criterios lógicos matemáticos de las relaciones uno a uno, uno a varios o varios a uno. Estas comprenden la forma en cómo se vinculan los elementos de dos tablas que corresponden dentro de un mismo género en el estudio, pero tienen dos temáticas distintas. Esto último, es clave para alcanzar una técnica de diseño eficiente, que permita una base de datos que enlace los datos en la misma forma. Tal es el caso del Sistema de Control de Ambiente y Temperatura, el cual básicamente comprende entre otros sistemas del Aire Acondicionado, se observa que, tal y como se ha señalado en apartado de clasificación de los Equipos de Aire acondicionado, que los equipos que lo constituyen, se encuentran a su vez clasificados en subsistemas con funciones particulares distintas, tales como la "ductería metálica" encargada de transferir el aire frío a los diversos ambientes, o el de la tubería encargada de llevar el agua helada a cada unidad manejadora de aire, que a su vez es producida desde los equipos enfriadores llamados técnicamente Chillers. Ahora bien, entre estos subsistemas se establecen relaciones dependientes de tipo operativo, en cuanto al objetivo de controlar la temperatura ambiental, pero que en sí mismos representan unidades operacionales independientes en cuanto a su propio funcionamiento si se les desvincula del objetivo ya señalado.

No obstante, es necesario determinar la forma en que se relacionan todos los elementos, primero en cada subsistema y luego con los subsistemas adyacentes, adicionalmente deben asociarse a estas relaciones, los eventos fortuitos o controlados que se presentaran y las acciones y registros que a partir de ello se generan. Todo esto debe quedar claramente reflejado en el diseño y elaboración de las tablas, de manera que pueda establecerse las relaciones lógicas (1 a 1; 1 a varios $(\infty)$; varios $(\infty)$ a 1 ; etc.) que se dan entre los elementos de dos subsistemas distintos mediante un mecanismo de enlace diseñado para tal propósito que permita a su vez, determinar la ruta de eventos con sus respectivos archivos de registro. 
Revista ALCONPAT, Volumen 1, Número 1, Enero-Abril 2011, Páginas 50 - 63

Las bases de datos relacionales permiten resolver en forma eficiente y amigable este objetivo. Antes que fueran desarrolladas las bases de datos relacionales, había que controlar las relaciones entre los archivos o tablas de forma manual. Por ejemplo: era necesario ubicar los datos del equipo en la tabla Equipos A/ACC y a continuación buscarse el registro coincidente con la tabla que lleva el control de las Solicitudes de Servicio de Mantenimiento para ese equipo en particular. Con un buen diseño las base de datos (Ramez 1997) relacionales resuelven este problema sin tenerse que preocupar por la forma en que deben enlazarse los datos, tal como se presenta en la Figura 1.

Mediante esta interacción es donde interviene la necesidad de alcanzar el buen diseño del código asignado a cada equipo, el cual es una pequeña y breve información incorporada a cada tabla, como la que se presenta en la Tabla 1, diseñada de forma tal que permite enlazar las tablas como por ejemplo los campos Identificación del Equipo (IDEquipo) con ID de la Solicitud de Servicio o el reporte de falla o la orden de trabajo a la cual diera origen. Estos códigos o campos de "enlace" en las tablas se denominan claves externas o ajenas.

Tabla 1. Formato de Planilla de Codificación

\begin{tabular}{|l|l|l|l|}
\hline $\begin{array}{l}\text { Museo de Arte } \\
\text { Contemporáneo } \\
\text { Dpto. De Mantenimiento }\end{array}$ & \multicolumn{2}{|l|}{ PLANILLA DE } & Formato $\mathbf{N}^{\circ}$ \\
CODIFICACIÓN & Hoja: \\
\hline Área: & Zona & $\begin{array}{l}\text { Responsable: } \\
\text { Responsable: } \\
\text { Firma: } \\
\text { Fecha: }\end{array}$ \\
\hline
\end{tabular}

\begin{tabular}{|l|l|}
\hline CÓDIGO & \\
\hline & \\
\hline & \\
\hline & \\
\hline & \\
\hline & \\
\hline & \\
\hline & \\
\hline & \\
\hline & \\
\hline & \\
\hline
\end{tabular}

En una base de datos (BD) bien diseñada, las claves externas dan como resultado una mayor eficiencia. Se puede controlar las claves externas relacionadas cuando se inicia el diseño de la correspondiente base de datos. Cuando se define las tablas en la BD, se debe enlazar la clave principal (código de identificación) con las claves ajenas para indicar a la BD la forma en que se tendrá que unir los datos cuando se necesita obtener información procedente de más de una tabla, tal como se presenta en la Figura2. 
Revista ALCONPAT, Volumen 1, Número 1, Enero-Abril 2011, Páginas 50 - 63

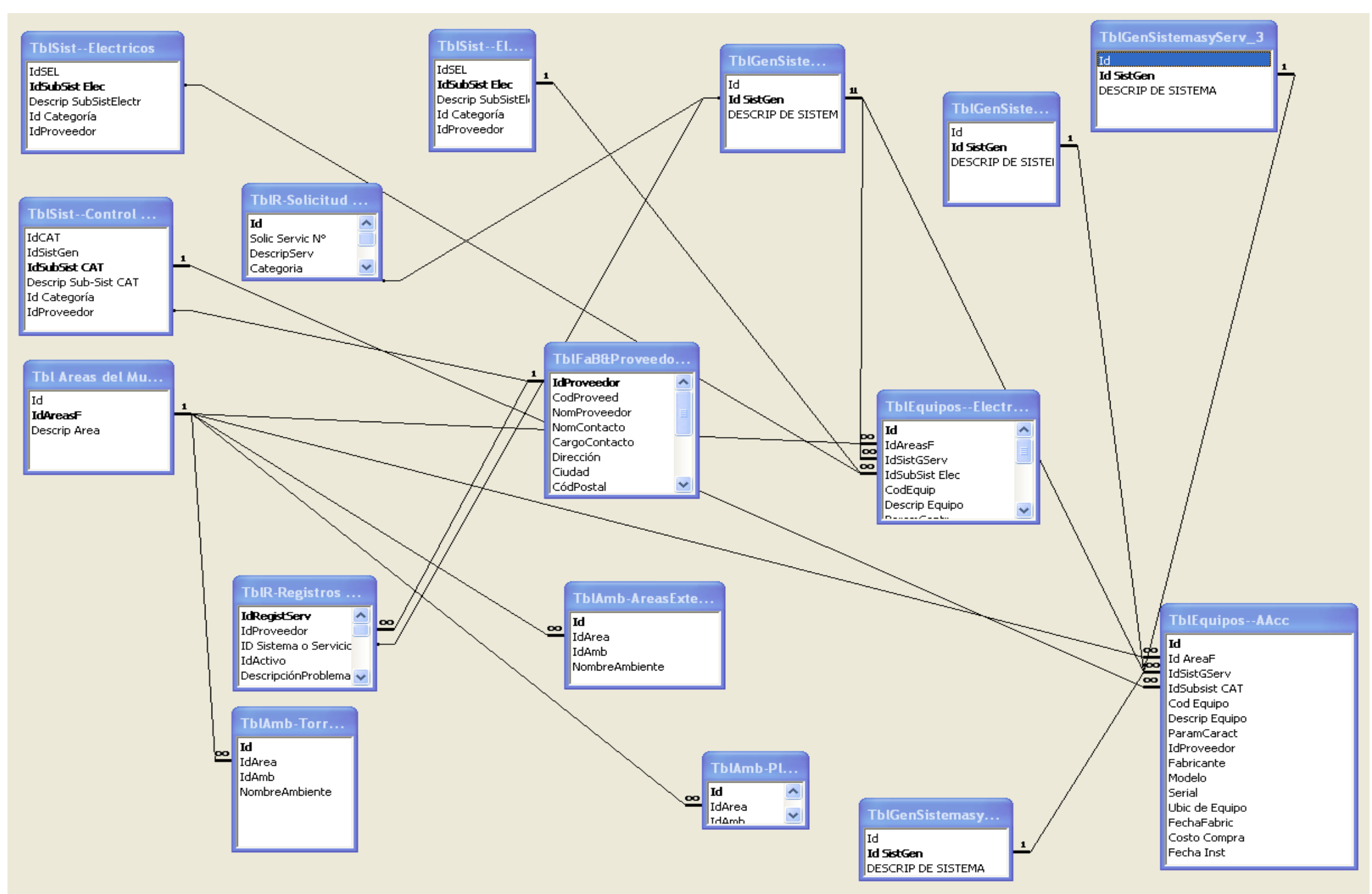

Figura 1. Relaciones entre las diferentes Tablas de la Base de Datos Generada para el Plan de Mantenimiento del Museo 


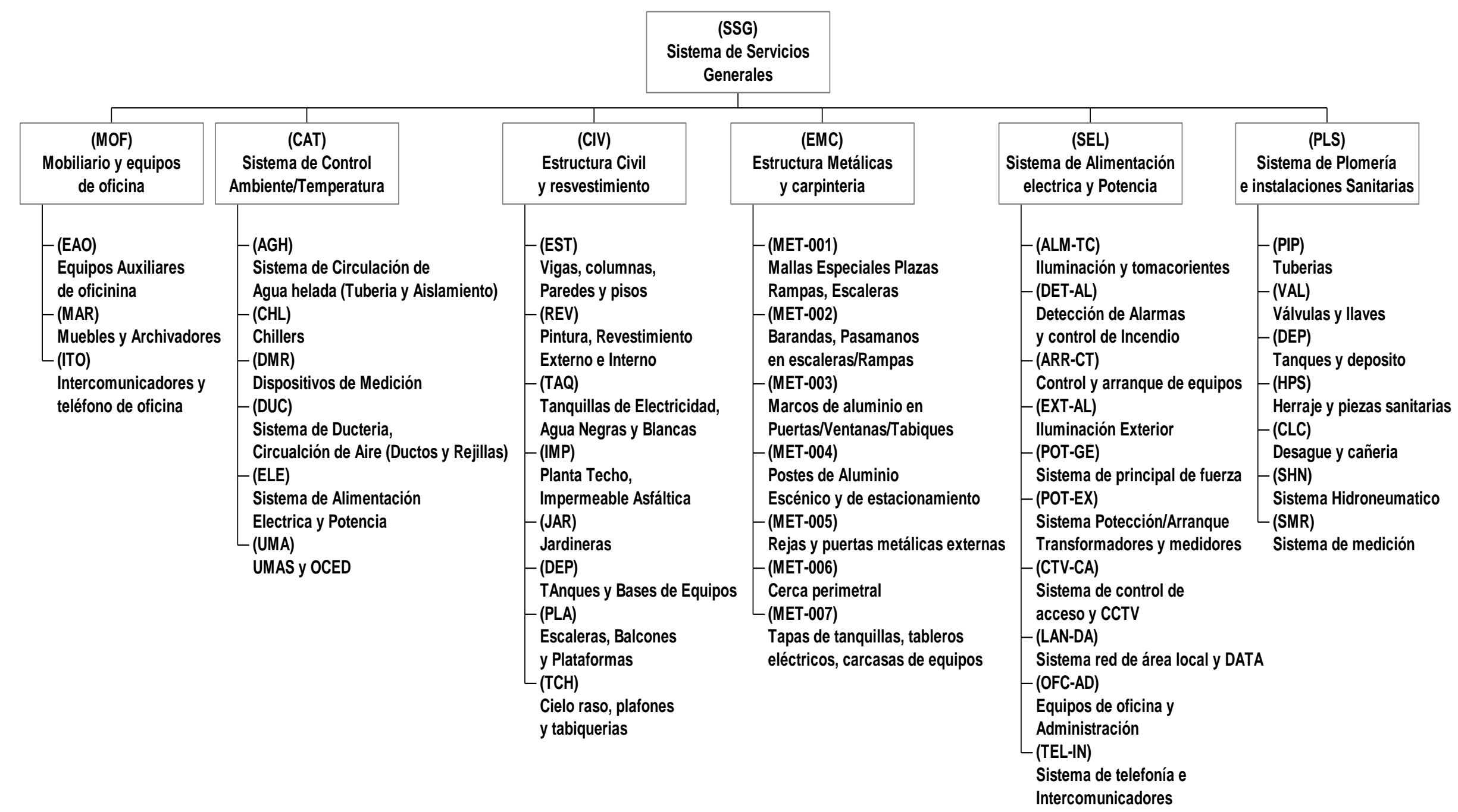

Figura 2. Esquema de Codificación para los diversos elementos de cada uno de los Subsistemas del Sistema de Servicios Generales 
En la mayoría de los casos, la relación eficiente entre dos tablas es uno a varios. Es decir, para cualquier registro de la Tabla 1, existen varios registros relacionados en la Tabla 2, pero para cualquier registro de la Tabla 2, existe solamente un registro coincidente en la Tabla 1. En el diseño de la BD del Museo se puede tener varios subsistemas pertenecientes, por ejemplo, al Sistema de Aire Acondicionado, a los cuales se encuentran asignados todos los equipos pertenecientes a cada subsistema. Un subsistema cualquiera tendrá asignados varios equipos que le pertenecen al mismo, pero un determinado equipo solo pertenecerá a un solo subsistema, tal como se representa en la Tabla 2.

El estudio ha tratado de determinar las particularidades y exigencias que tiene el Museo para el prestar el servicio al cual está destinada, así como sus elementos más relevantes en cuanto infraestructura y servicios.

Se pretende primero resaltar la necesidad de afrontar el tema del mantenimiento del Museo según los criterios de la Gerencia de Mantenimiento moderna, mediante el uso de una estructura de gestión de la información, exigente, que de forma flexible, ágil, eficaz y a bajo costo, ayude a elaborar el análisis y la planificación del mantenimiento en forma eficiente en este tipo de instituciones. Parar ello se han elaborado en primer lugar clasificaciones de los elementos que intervienen o conforman en el museo, formatos que ayuden a recopilar la información que debe incorporarse a la estructura de gestión de la información (Tablas 3, 4, 5 y 6, Figura 3).

\section{RESULTADOS Y DISCUSIÓN DE RESULTADOS}

Se han dado en el presente trabajo los criterios y metodologías que permiten la elaboración y uso de las bases de datos y sus técnicas diseño como recurso poderoso para procesar grandes volúmenes de información en forma rápida y eficaz, usando el computador como poderosa herramienta y cuyo uso actualmente masificada mediante soluciones de software que facilitan la ejecución casi doméstica de procesos automatizados de control manejo y supervión pero no por eso menos sofisticados en sus resultados inmediatos.

El aporte más destacado al llevar a cabo la elaboración de una BD consiste, en que el mismo exige primeramente el levantamiento y registro de todos los activos en equipos y sistemas de la edificación museística, facilitando la tarea de llevar el registro histórico y control de los diversos eventos que van presentándose en estos últimos, durante la vida misma de la edificación, así como de sus sistemas de apoyo y servicios. Esto constituye el soporte básico para la elaboración de las metodologías y procedimientos requeridos para un plan de mantenimiento.

Ciertamente, el registro histórico y estadístico de los eventos asociados a cada una de las áreas de sistemas y servicios en cualquier edificación o infraestructura (sea esta museística o de cualquier tipo) genera un gran volumen de información que puede llegar a desbordar la capacidad de procesamiento de la misma. Es aquí donde las bases de datos presentan su mayor potencial de uso como herramienta, ya que permiten organizar, clasificar, jerarquizar y evaluar los aspectos y parámetros esenciales que deben ser contralados en un plan de mantenimiento de modo inmediato y preciso, de forma breve y confiable y dirigida a los aspectos esenciales del mismo.

Como consecuencia de lo anterior, se lleva a cabo la optimización en el uso de los recursos disponibles (humanos, materiales y la planificación asociada de trabajos y actividades requeridos así como de los costos económicos, amen de los ahorros de tiempo implícitos por la implementación oportuna de planes de mantenimiento preventivos).

Actualmente existen desarrollos de software en el mercado orientados hacia el área de mantenimiento, de cierta importancia, pero en su mayor parte son sistemas desarrollados bajo una estructura cerrada, dirigidos básicamente hacia la industrias o empresas productoras de bienes y consumos y aunque quizá algunos de ellos pueden adaptarse a los requerimientos de instituciones 
Revista ALCONPAT, Volumen 1, Número 1, Enero-Abril 2011, Páginas 50 - 63

tales como los museos, exigen de cierta inversión de dinero, tiempo y entrenamiento que en la mayoría de los casos resulta oneroso y poco práctico.

Tabla 2. Distribución de Equipos Principales de Cada Sistema o Servicio

\begin{tabular}{|c|c|}
\hline Sistemas de Control Ambiental y Temperatura & 001 \\
\hline 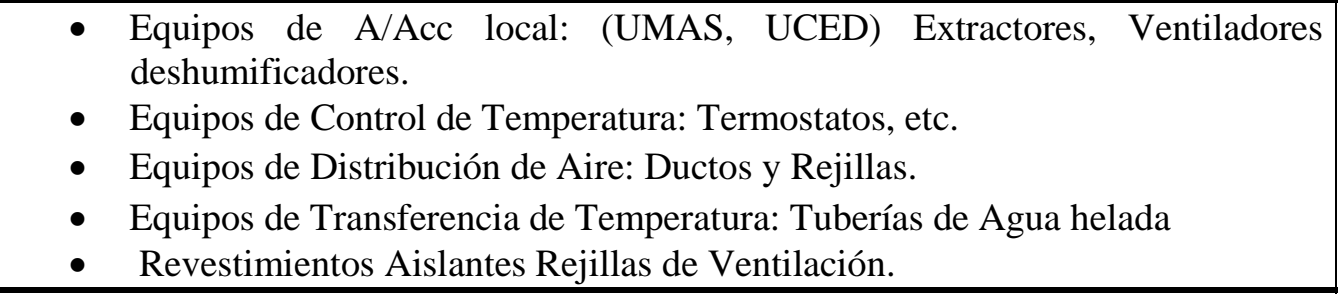 & \\
\hline Servicio de Aguas Blancas e Instalaciones Sanitarias & 002 \\
\hline $\begin{array}{l}\text { - Tanques de Agua } \\
\text { - Tuberías } \\
\text { - Válvulas y Llaves } \\
\text { - Salas y Equipos Sanitarios } \\
\text { - Sistema Hidroneumático }\end{array}$ & \\
\hline Sistemas y Equipos de Iluminación y Tomacorrientes & 003 \\
\hline 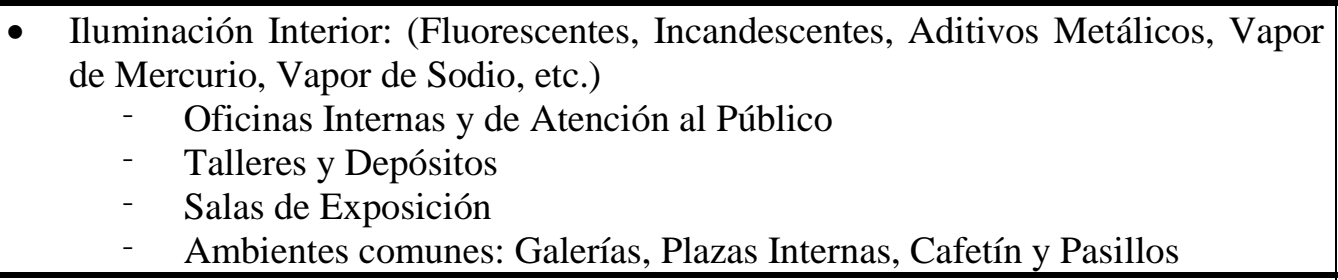 & \\
\hline \multicolumn{2}{|l|}{ Sistema de Alumbrado Exterior } \\
\hline $\begin{array}{l}\text { - Iluminación Exterior: (Fluorescentes, Incandescentes, Aditivos Metálicos, } \\
\text { Vapor de Mercurio, Vapor de Sodio, etc.) } \\
\text { - Fachada } \\
\text { - Estacionamientos } \\
\text { - Vigilancia }\end{array}$ & \\
\hline Estructuras Metálicas & 003 \\
\hline $\begin{array}{l}\text { - } \text { Barandas de Rampas y Escaleras } \\
\text { - Puertas Metálicas y Romanillas } \\
\text { - Rejas y Protecciones Metálicas } \\
\text { - Armarios y Estanterías } \\
\end{array}$ & \\
\hline Estructura civil & 004 \\
\hline $\begin{array}{l}\text { - Revestimientos de Obra Limpia } \\
\text { - } \quad \text { Impigas y Columnas } \\
\text { - Paredes y Mamposterías } \\
\text { - Tabiquería } \\
\text { - Cielos Rasos } \\
\text { - Puertas y Ventanas de Vidrio } \\
\end{array}$ & \\
\hline Equipos de Mando y Operación de Sistemas y Dispositivos Eléctricos & 005 \\
\hline $\begin{array}{ll}\text { - } & \text { Tableros Eléctricos } \\
\text { - } & \text { Sistema de Detección de Incendio }\end{array}$ & \\
\hline
\end{tabular}


Revista ALCONPAT, Volumen 1, Número 1, Enero-Abril 2011, Páginas 50 - 63

- Sistema de Seguridad Interna: Control de Acceso, CCTV, etc.

Mobiliarios y Equipos de Oficina

- Escritorios, Archivadores y Estanterías

- Computadoras, fotocopiadoras, máquinas de escribir,

- Sillas, mostradores, etc.

Tabla 3. Clasificación de Áreas del Museo

- PLANTA FISICA (Edificio Central)

Salas de Exposición

Zonas Administrativas y de Oficinas

Servicios al Público (CIDAC, Cafetín, auditorios, etc)

- TORRE ADMINISTRATIVA

Laboratorio de Restauración y conservación

Oficinas de supervisión y seguridad

- AREAS EXTERIORES.

Jardines y Estacionamientos

- SERVICIOS GENERALES Y DEPOSITOS

Sala de Equipos de Aire Acondicionado

Depósitos de Obras permanentes y obras en tránsito

Rampas y Puertas de Acceso laterales al edificio

Techo.

Ciertamente, el planteamiento anterior no excluye en nuestro caso de una cierta preparación para acometer la tarea de desarrollo e implementación de un plan de mantenimiento usando el computador como herramienta de soporte, pero lo que se quiere en este caso, es la capacidad de involucrar para el proceso mismo del mantenimiento de instituciones museísticas, aquellos recursos que debido a su bajo costo, relativa sencillez y uso cada vez más extenso estén unidos a una metodología de seguimiento, gestión y procesamiento de la información estandarizada, que a su vez permita la adaptación tanto de la herramienta de trabajo a las necesidades del proceso de mantenimiento como del recurso humano que debe interactuar con ella.

No cabe duda que una buena planificación del mantenimiento ayudará al buen desempeño del servicio que presta el museo, si se tienen como ya se ha insistido en este trabajo con los recursos necesarios cualquiera que sea la naturaleza de los mismos, pero el recurso financiero junto al apoyo de una estructura administrativa eficaz es especial y de particular importancia, tanto por la organización del museo como por parte de los organismos oficiales y privados que le sirven de patrocinio, pues ello garantiza el disfrute estético y educativo por el mayor tiempo posible del patrimonio artístico y el acceso del público a un precio razonable a las colecciones y obras que de otra forma serian inaccesibles.

En nuestro país es necesario llevar una tarea de sensibilización del alcance y los múltiples beneficios educativos y de formación en la cultura de los pueblos, donde las instituciones museísticas están llamados a realizar y en este sentido diseñar aportes en todos los ámbitos de la sociedad, sean estos de naturaleza social, política, técnica o financiera de forma que se pueda proyectar con el mayor de los alcances los grandes beneficios culturales que estas generan en las sociedades que les dan cabida. 
Tabla 4. Formato de Planilla de Diagnóstico.

\begin{tabular}{|l|l|l|l|}
\hline $\begin{array}{l}\text { Museo de Arte Contemporáneo } \\
\text { Dpto. De Mantenimiento }\end{array}$ & \multicolumn{2}{|c|}{ SITUACIÓN ACTUAL Y DIAGNÓSTICO } & $\begin{array}{l}\text { Formato No } \\
\text { Hoja: }\end{array}$ \\
\hline Área: & Totales & Responsable: \\
\cline { 2 - 4 } & Inspeccionados & $\begin{array}{l}\text { Firma: } \\
\text { Fecha: }\end{array}$ &
\end{tabular}

\begin{tabular}{|c|c|c|c|c|c|c|c|c|c|c|c|c|c|c|}
\hline \multirow[b]{2}{*}{ Descripción } & \multicolumn{12}{|c|}{ CONDICIÓN } & \multicolumn{2}{|c|}{ INTERVENCIÓN } \\
\hline & SA & SU/IN & VI & GR & RO & DE & NOC & IN & PI & HU/FI & $\mathrm{NE}$ & $\mathbf{B}, \mathbf{R}, \mathbf{M}$ & Inmediata & \begin{tabular}{|l|} 
Parada \\
Programada
\end{tabular} \\
\hline \multicolumn{15}{|l|}{ Paredes } \\
\hline \multicolumn{15}{|l|}{ Vigas } \\
\hline \multicolumn{15}{|l|}{ Ventanas } \\
\hline \multicolumn{15}{|l|}{ Vidrios } \\
\hline \multicolumn{15}{|l|}{ Puertas } \\
\hline \multicolumn{15}{|l|}{ Techos } \\
\hline \multicolumn{15}{|l|}{ Cielo Raso } \\
\hline \multicolumn{15}{|l|}{ Piso } \\
\hline \multicolumn{15}{|l|}{ Cerradura } \\
\hline \multicolumn{15}{|l|}{ Lámparas } \\
\hline \multicolumn{15}{|l|}{ Inst. Eléctricas } \\
\hline \multicolumn{15}{|l|}{ Enchufes } \\
\hline \multicolumn{15}{|l|}{ Interruptores } \\
\hline \multicolumn{15}{|c|}{ Sist. De Vent y A/Acc } \\
\hline \multicolumn{15}{|l|}{$\begin{array}{c}\text { Línea Telefónica } \\
\text { Teléfono } \\
\end{array}$} \\
\hline $\begin{array}{c}\text { Reja Puertas } \\
\text { Rejas Ventanas }\end{array}$ & & & & & & & & & & & & & & \\
\hline
\end{tabular}

SA: Satisfactorio; SU: Suelto; IS: Inseguro; VI: Vibración; GR: Grietas; RO: Roto; DE: Deformado; NOC: No Opera Correcto; IN: Inadecuado; PI: Pintura; HU: Humedad; FI: Filtración; NE: No Existe; B: Bueno; R: Regular; M: Malo 
Tabla 5. Formato de Planilla de Inventario.

\begin{tabular}{|l|l|l|}
\hline $\begin{array}{l}\text { Museo de Arte } \\
\text { Contemporáneo } \\
\text { Dpto. De Mantenimiento }\end{array}$ & PLANILLA DE INVENTARIO & $\begin{array}{l}\text { Formato } \mathbf{N}^{\circ} \\
\text { Hoja: }\end{array}$ \\
\hline & $\begin{array}{l}\text { Semana: } \\
\text { Sección: } \\
\text { Ejecutores: }\end{array}$ & $\begin{array}{l}\text { Responsable: } \\
\text { Firma: } \\
\text { Orden de Trabajo } \\
N^{\text {Fecha: }}\end{array}$ \\
\hline \multicolumn{2}{|l|}{ Descripción } & \\
\hline
\end{tabular}

Tabla 6. Formato de Orden de Trabajo

\begin{tabular}{|c|c|c|}
\hline $\begin{array}{l}\text { Museo de Arte } \\
\text { Contemporáneo } \\
\text { Dpto. de Mantenimiento }\end{array}$ & ORDEN DE TRABAJO & $\begin{array}{l}\text { Formato } \mathrm{N}^{\circ} \\
\text { Hoja: }\end{array}$ \\
\hline & \begin{tabular}{|l} 
Semana: \\
Sección:
\end{tabular} & Responsable: \\
\hline $\begin{array}{l}\text { Orden de Trabajo } \\
\mathrm{N}^{\circ}\end{array}$ & Ejecutores: & $\begin{array}{l}\text { Firma: } \\
\text { Fecha: }\end{array}$ \\
\hline
\end{tabular}

\begin{tabular}{|l|l|l|l|l|l|l|l|l|l|l|}
\hline Código & L & M & M & J & V & S & COMENTARIO & MATERIAL & H/H & $\begin{array}{c}\text { COSTO } \\
\text { TOTAL }\end{array}$ \\
\hline & & & & & & & & & & \\
\hline & & & & & & & & & & \\
\hline & & & & & & & & & & \\
\hline
\end{tabular}

AREAS Y AMBIENTES DEL MACZUL

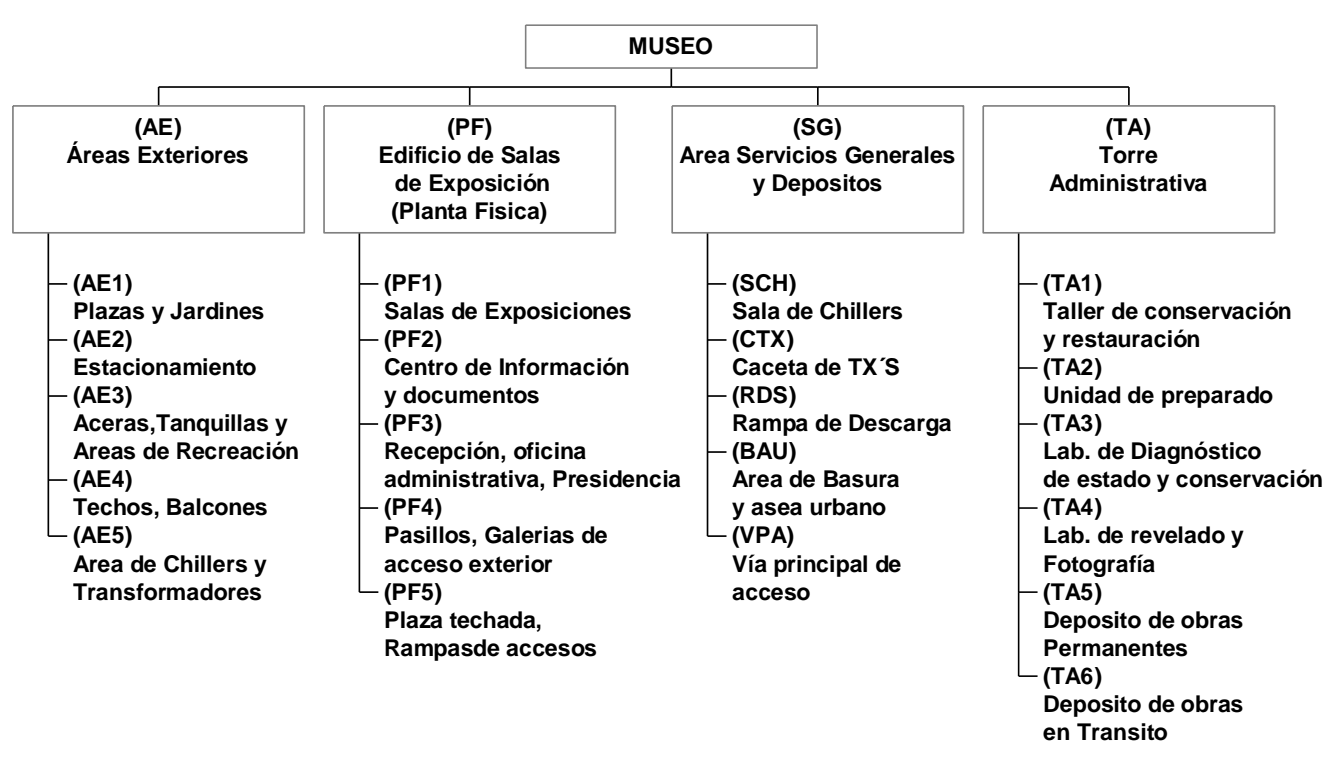

Figura 3. Distribución de ambientes en Áreas del Museo (En paréntesis prefijo codificación) 
Finalmente, como ya se ha señalado anteriormente, el éxito de un plan de mantenimiento no solo depende de eficiente y sofisticado que el mismo sea, sino en mayor medida del apoyo que los encargados de llevarlo a cabo reciban, tanto de los responsables directos de su elaboración e implementación, como de los cuadros gerenciales y ejecutivos de la institución, los cuales deberán prever entre otros aspectos, el apoyo logístico y presupuestario que garantice su implementación oportuna y adecuada.

Tal como lo señala en su obra A. Perozo (1999) en su texto de enseñanza "Gerencia de Mantenimiento" "Muchas personas, incluyendo profesionales, consideran el termino Sistema Gerencial de Mantenimiento como un sistema Computarizado, en ese particular, es un error considerar el término en un sentido tan restringido, sino más bien como un sistema total, conformado por políticas, organización, sistemas y procedimientos. El sistema Computarizado es solo una parte del Sistema Total". Lo anterior señala la necesidad de incorporar tanto desde el punto de vista operativo por parte del responsable como desde la concepción que los cuadros directivos tengan del proceso de mantenimiento, todos los elementos que vaya exigiendo la dinámica de su implementación, los cuales van más allá de la efectividad o no del Plan de Mantenimiento, sino inclusive de su capacidad de insertar en la conciencia de todos los involucrados (tanto del personal que labora en la institución como del público al cual están dirigidos los servicios del museo) la importancia llevar los esfuerzos que sean necesarios para garantizar la preservación del patrimonio artístico y cultural de las obras que se exponen y que también representan junto a los museos mismos, la identidad cultural y el legado de una nación o pueblo a las generaciones por venir.

\section{CONCLUSIONES}

Para alcanzar racionalmente la preservación en el mayor tiempo posible de las instalaciones del Museo y por ende de las colecciones artísticas bajo su custodia, va depender en gran medida, del apoyo tanto gerencial como presupuestario a las múltiples y diversas actividades que se hallan implícitas en la aplicación práctica del presente modelo de gestión del mantenimiento.

De continuarse con la improvisación y fallas de planificación se estará afectando la norma habitual en el desarrollo del mantenimiento con el incremento asociado en el riesgo de situaciones de daño y deterioro de los bienes culturales y patrimonio artístico bajo custodia de la institución.

Del análisis realizado puede inferirse que en el proceso de desarrollo de un Plan de Mantenimiento orientado a facilitar el desempeño funcional y operativo de un museo debe cubrirse como mínimo los siguientes aspectos:

1. Levantamiento, evaluación y diagnóstico de las condiciones físicas y de seguridad existentes de las salas o ambientes en las cuales pueden permanecer, expuestas o almacenadas las distintas obras de arte

2. Poner énfasis en garantizar en el mayor grado posible, las óptimas condiciones ambientales (luz, temperatura, humedad y seguridad contra actos vandálicos) de las salas y dependencias en las cuales se realiza la exposición o almacenamiento de las obras, previendo el equipamiento de los sistemas faltantes que sean necesarios para garantizar la preservación y buen estado de las obras artísticas en el tiempo

3. Conocimiento cabal la dinámica de los sistemas y servicios y de cómo se ven afectados según el ámbito y circunstancias locales de la edificación y el ambiente que la rodea. Llevar a cabo un levantamiento físico de los materiales y equipos existentes y/o que deben ser adquiridos y con ello una recopilación documental de los requerimientos (manuales, catálogos procedimientos de operación y mantenimiento, entre otros) para el cuidado y buen funcionamiento de los mismos. 
Revista ALCONPAT, Volumen 1, Número 1, Enero-Abril 2011, Páginas 50 - 63

4. Diagnóstico oportuno y jerarquizado de los riesgos de deterioro a los cuales se puede ver expuestas las obras según su naturaleza y características propias, así como de las exigencias presentes de las áreas de exposición y de los sistemas existentes. Las obras deberán ser clasificadas en función a sus características propias y ser trasladadas a las áreas o dependencias que garanticen las condiciones externas señaladas en el primer párrafo

5. Recopilación, organización y clasificación de los procedimientos operativos del mantenimiento según sus costos, complejidad, frecuencia de ejecución, para evaluar la trascendencia e impacto de las acciones de mantenimiento en la preservación o el deterioro del patrimonio artístico de manera que esto permita fijar criterios de selección y prioridades para la planificación del mismo, así como la cuantificación de los costos asociados

6. Registro histórico y estadístico de tasa de eventos de fallas, ordenes de servicio y puesta en marcha de los sistemas y equipos de mayor impacto tanto en los costos como en mantenimiento de las condiciones de preservación y conservación de las obras

7. Definición, Coordinación y planificación de las tareas y actividades a partir de las evidencias que se obtengan del análisis de los resultados arrojados por la base de datos y las circunstancias particulares de la institución.

8. Finalmente, es fundamental que todo lo anterior sea sustentado mediante la definición institucional de una sólida política gerencial de mantenimiento que contemple: apoyo gerencial y presupuestario a estructura y organización del Departamento o estructura de mantenimiento del museo, estímulos e incentivos de permanencia y adiestramiento al personal responsable del departamento de mantenimiento; previsiones oportunas de apoyo en recursos humanos, materiales, técnicos y económicos que, a partir de una gestión eficaz necesaria, permita extender la vida útil de las instalaciones y equipos, , y que redunde en el futuro inmediato en obtener los resultados deseados con la menor inversión. (G Salas R, A Algarra y otros 1981).

\section{BIBLIOGRAFIA}

Consejo Nacional de la Cultura (CONAC) (1992). Normas para el Diseño y Preservación de Museos. La Conservación del Museo.- La Infraestructura Física de Museos. Caracas, Venezuela. Consejo Internacional de Museos.- II Seminario sobre patrimonio Cultural "Cultura, Museos y Política Económica" Buenos Aires, 19-23 Octubre 1.993.- Código de Ética y Deontología Profesional del ICOM.- Cáp. II: Deontología de las Instituciones:

Figueroa S. S J Abreu L R. (2002) Modelo Gerencial para implementar un sistema de Mantenimiento de las Instalaciones y Bienes de las Instituciones Universitarias (Oct. 1998) Universidad de los Andes Mérida 5101. Trabajo presentado en las X Jornadas Científico Técnicas de Ingeniería.

Lucena I. (1999) Conservadora de obras de Arte. Causas de deterioro en las colecciones por la inadecuada proyección en la concepción de un museo. Artículo presentado para publicación de la revista del museo.

Fundación Museo de Arte Contemporáneo del Zulia (2000). Consejo Nacional de la Cultura, Universidad del Zulia. El Infinito Canto de Este Sol, Arte y Cultura del Zulia, 1780-1998. J\&Eme. Editores, S.A., Centro de Bellas Artes, Ateneo de Maracaibo.

MACZUL. Museo de Arte Contemporáneo del Zulia. (1999). Coordinación de Conservación y Restauración. Curso de Conservación Preventiva. Maracaibo, Venezuela

Morrow C. (1984) Manual de Mantenimiento Industrial. Tomo 2 Cía. Editorial Continental, S.A. de C. V. México DF. México. Novena Edición.

Plan integral de mantenimiento preventivo en las instalaciones del museo arte... 
Revista ALCONPAT, Volumen 1, Número 1, Enero-Abril 2011, Páginas 50 - 63

Nava Aranguren. J, D. (1996) Teoría del Mantenimiento, Definición y Organización. Universidad de los Andes.- Consejo de Publicaciones. Mérida. Venezuela.

Perozo A. (1999) Gerencia de Mantenimiento. Universidad del Zulia. Texto guía de la cátedra de Gerencia de Mantenimiento. Maracaibo, Octubre de 1999.

Provenzali L. (2000) Editorial IPCOMENTARIOS. Revista del Instituto del Patrimonio Cultural. Año 1 Numero 6

Ramez E., Shamkant B. N. (1997) Sistemas de Bases de Datos. Conceptos Fundamentales. Addison Wesley Iberoamericana. 1997. Versión en español de Roberto Escalona García. México D.F.

Stewart H. V. M. (1968) El Departamento de Mantenimiento en la Empresa. Traducción por José Ignacio Galdeano. $2^{\text {a }}$ Edición. Ediciones Deusto. Bilbao, España.

Salas R, G y otros (1.981) Tema II.1 El Mantenimiento de la Infraestructura Física e Instalaciones Médico-Asistenciales. Primeras Jornadas Nacionales de Mantenimiento. Caracas, 17 al 20 de Marzo de 1981. 